\title{
Cerebellar Syndrome Revealing a Giant Postero Inferior Cerebellar Artery Aneurysm: A Case Report and a Review of Literature
}

\author{
Oumar Diallo ${ }^{*}$, Maiga Abdoulaye Hima², Beketi Katanga Anthony ${ }^{3}$, Thioub Mbaye ${ }^{4}$, \\ Mahamadou Dama1, Oumar Coulibaly'1, Daouda Sissoko', Alpha Singuepire5, \\ Drissa Kanikomo6, Seydou Boubakar Badiane4 \\ ${ }^{1}$ Hospital of Mali, Bamako, Mali \\ ${ }^{2}$ Hospital of Niamey, Niamey, Niger \\ ${ }^{3}$ University of Kara, Kara, Togo \\ ${ }^{4}$ University Checkh Anta Diop, Dakar, Senegal \\ ${ }^{5}$ Regional Hospital of Segou, Ségou, Mali \\ ${ }^{6}$ Hospital Gabriel Touré, Bamako, Mali \\ Email: *tiecourala2003@yahoo.fr
}

How to cite this paper: Diallo, O., Hima, M.A., Anthony, B.K., Mbaye, T., Dama, M., Coulibaly, O., Sissoko, D., Singuepire, A., Kanikomo, D. and Badiane, S.B. (2021) Cerebellar Syndrome Revealing a Giant Postero Inferior Cerebellar Artery Aneurysm: A Case Report and a Review of Literature. Case Reports in Clinical Medicine, 10, 153-159. https://doi.org/10.4236/crcm.2021.106019

Received: April 30, 2021

Accepted: June 7, 2021

Published: June 10, 2021

Copyright $\odot 2021$ by author(s) and Scientific Research Publishing Inc. This work is licensed under the Creative Commons Attribution International License (CC BY 4.0).

http://creativecommons.org/licenses/by/4.0/ (c) (i) Open Access

\begin{abstract}
Context: Cerebellar syndrome caused by disturbances of balance and coordination is not an uncommon neurological disorder. It has varied etiologies usually caused by tumor processes or suppurative collections. Vascular causes remain very rare, especially when it is a giant aneurysm of PICA representing $1 \%$ of intracranial aneurysms. Treating giant PICA aneurysms is a very difficult task for neurosurgeons because the surgical dissection can lead to severe damage due to the intimate relationship of PICA with the brainstem or nerve structures. We report a case of giant PICA aneurysm responsible for cerebellar syndrome successfully treated with surgery. The objective of this work is to draw the attention of practitioners to this unusual cause which can lead to diagnosis wandering and a lack of planning at the time of management. Case report: A 65 years old hypertensive patient was seen for a progressive disturbance of balance and walking disorder, but worsened in the last three months with no notion of fever. On admission, the patient was lucid, oriented in time and space and presented with static and kinetic cerebellar syndrome. Brain CT-scan without and with contrast revealed a tissular mass in the posterior fossa suggesting a tumor process, however, CT angiography showed a giant aneurysm of the PICA after reconstruction. A careful microdissection by a sub-occipital approach was decided. Opening the large cistern made it possible to visualize the aneurysm sack surrounded by a yellowish gliosis. The reclining and microdissection revealed the neck of the aneurysm, which was
\end{abstract}


clipped to exclude the giant aneurysm in block. Postoperative follow-up was simple with progressive improvement in the cerebellar syndrome and walking over three months. Conclusion: Giant aneurysm of the PICA is rare. The localization in the posterior cerebral fossa can be confusing. Microsurgery gives a good result.

\section{Keywords}

Giant Aneurysm, PICA, Cerebellar Syndrome, CT Angiography

\section{Introduction}

PICA aneurysms represent $1 \%$ of all intracranial aneurysms [1] and giant aneurysms defined by their large diameter greater than $25 \mathrm{~mm}$ represent $2 \%$ to $3 \%$ of intracranial aneurysms [2] [3]. The localization at the Ponto-cerebellar angle leading to a cerebellar syndrome (a static and kinetic cerebellar syndrome leading to disturbances in balance and coordination of movements) is a rare clinical representation which can be confusing and whose management remains a challenge [4]. Microdissection clipping can cause serious neurological complications due to the proximity of the aneurysm sack to the brainstem and cranial nerves. We present a case of giant PICA aneurysm that resulted in cerebellar syndrome successfully treated by clipping and a review of the literature.

\section{Clinical Case}

A 65 years old patient with a history of controlled hypertension on amlodipine seen for problem with balance and walking. Medical story was consistent with progressive disabling headaches evolving for three years and complicated by right unilateral perceptual hypoacusis, balance and coordination disorders three months ago. A kinetic and static cerebellar syndrome was shown on physical examination. Brain Ct-scan without and with injection of contrast revealed an expansive process in the posterior cerebral fossa at the level of the Ponto-cerebellar angle with a double central hyperintense component surrounded by a less dense peripheral crown of $36.5 \mathrm{~mm}$ by $27 \mathrm{~mm}$, suggesting a tumor or vascular process (Figure 1). CT angiography revealed a giant aneurysm of the distal portion of the PICA after reconstruction (Figure 2). The patient did not agree with the surgery at the beginning. She left the hospital and returned two weeks later. After the worsening of the neurological signs and the anesthesiology consultation, Clipping surgery was decided for sub-occipital approach. The spinous of the first cervical vertebra was removed and the foramen magnum was exposed and opened completely. The dura mater was cut large and the PICA was observed then a thorough dissection and opening of the large cistern on the right side were performed. A yellowish scarring gliosis was seen serving as a cleavage plane surrounding the non-pulsatile aneurysm sac, indicating a micro-bleeding in the past. The reclining of the bag made it possible to see an anterior collar 




Figure 1. Brain CT-scan visualizing the giant aneurysm.

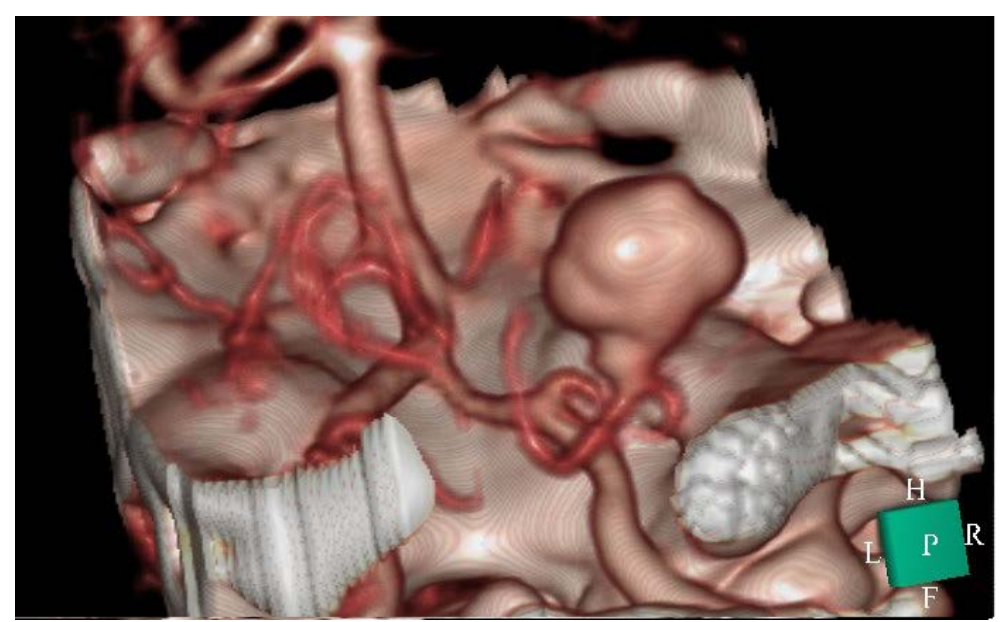

Figure 2. CT angiography visualizing the giant PICA aneurysm.

accessible by clipping. The aneurysm was excluded in block without retraction of the nerve structures by placing a medium right yasargil clip (Figure 3) and the sack resected in block (Figure 4). The postoperative treatment was straightforward, in particular she did not present with any additional neurological deficit or cranial nerve paralysis. The follow up brain scan showed the total exclusion of the aneurysm (Figure 5). The patient was put on physical therapy and tree months after discharge. After the third month, she had fully recovered and was independent. The patient was programmed for a follow-up with a CT-scan every year. 


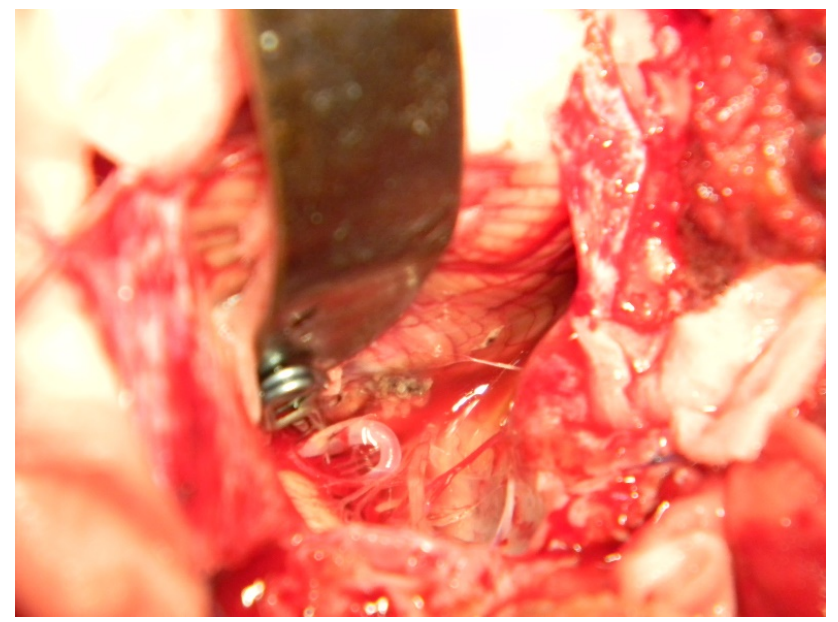

Figure 3. Intraoperative image view after the aneurysm is removed.

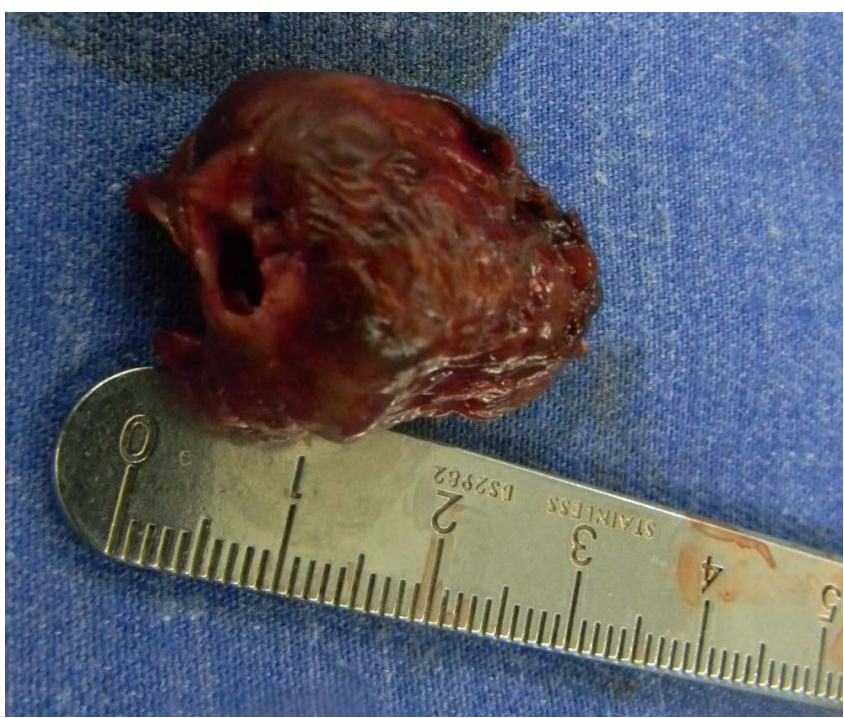

Figure 4. Photography of the giant aneurysm after surgical removal.

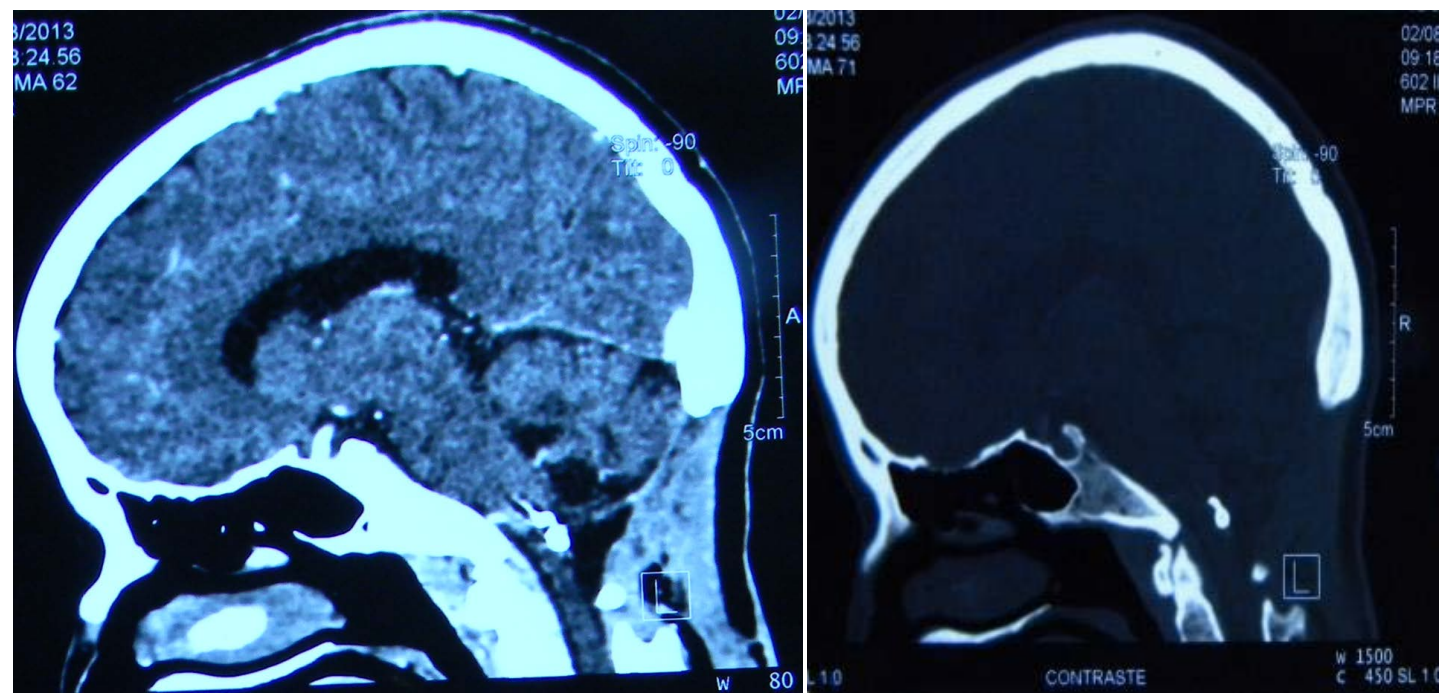

Figure 5. Postoperative brain CT-scan showing the total removal of the aneurysm three years after surgery. 


\section{Discussion}

The incidence of PICA aneurysms is $0.5 \%$ to $3 \%$ and giant aneurysms account for $3 \%$ to $5 \%$ of intracranial aneurysms [2] [3]. About (15\%) of these aneurysms are of carotid origin [5]. Localizations of these aneurysms at the level of the PICA in its distal portion are rare and represent $30 \%$ of aneurysms in PICA [6]. They can lead to paralysis of the cranial nerves or suffering of the cerebellar tissue [7] as observed in our patient. Slow progression and mass syndrome have been responsible for the coordination impairment. The brain CT-scan made it possible to evoke the diagnosis which was confused with a tumor process brain CT-angiography with the reconstructions visualized the giant aneurysm. Cerebral angiography remains the most important diagnosis tool, "the gold standard" [8], but was not performed in our patient because of its unavailability in the regional health center. The open surgical indication was retained and was the only therapeutic choice due to the unavailability of other methods. The choice of techniques and methods is controversial because each method has its advantages and disadvantages [9]. Several authors believe that surgical clipping brings better results [4] [5] [10] [11] and the sub-occipital route the most used [12]. In our case, this method made it possible to clip the aneurysm and exclude the aneurysmal sac which was compressing the cerebellum. Cranial nerve palsy and cerebellar infection are frequent and usual complications of PICA surgery, it has been reported that $47 \%$ of IX and X palsy and $22 \%$ of patients will continue to have dysphagia one year after surgery [13]. An understanding of the anatomy of this artery is essential [9] [14]. The mixed nerves were below the giant aneurysm and were not damaged, which explains the absence of aggravation or paralysis of the cranial nerves, postoperatively. Morbidity and mortality associated with microsurgery are relatively high, and endovascular embolization treatment is associated with several complications [4], Given the complications related to surgery and the limitations of endovascular treatment, it is necessary to consider other methods in the future, such as bypass and new bypass stents such as bypass PICA to PICA, occipital artery to PICA [10] [15] [16].

\section{Conclusion}

The PICA aneurysm is rare, even rarer when it is localized in the posterior cerebral fossa. Surgery prevents re-bleeding by clipping the aneurysm and also relieves the mass syndrome on the nervous structures by excluding the aneurysm.

\section{Conflicts of Interest}

The authors declare no conflicts of interest regarding the publication of this paper.

\section{References}

[1] Dammers, R., Krisht, A.F. and Partington, S. (2009) Diagnosis and Surgical Management of Extracranial PICA Aneurysms Presenting through Subarachnoid Hae- 
morrhage: Case Report and Review of the Literature. Clinical Neurology and Neurosurgery, 111, 758-761. https://doi.org/10.1016/j.clineuro.2009.05.012

[2] Choi, I.S. and David, C. (2003) Giant Intracranial Aneurysms. Development, Clinical Presentation and Treatment. European Journal of Radiology, 46, 178-194. https://doi.org/10.1016/S0720-048X(03)00090-1

[3] Lawton, M.T. and Spetzler, R.F. (1998) Surgical Strategies for Giant Intracranial Aneurysms. Neurosurgery Clinics of North America, 9, 725-742. https://doi.org/10.1016/S1042-3680(18)30225-0

[4] Zhu, J., Yin, L., Che, Y., Liu, Z., Qi, X., Zhou, K., Zheng, B., Pan, E. and Chen, J. (2020) Treatment of a Giant Complicated Distal Posterior Inferior Cerebellar Artery Aneurysm: A Case Report and Literature Review. Experimental and Therapeutic Medicine, 20, 395-400. https://doi.org/10.3892/etm.2020.8724

[5] Chen, J., Zhu, J., He, J., Wang, Y., Chen, L., Zhang, C., Zhou, J. and Yang, L. (2016) Ultra-Early Microsurgical Treatment within $24 \mathrm{~h}$ of SAH Improves Prognosis of Poor-Grade Aneurysm Combined with Intracerebral Hematoma. Oncology Letters, 11, 3173-3178. https://doi.org/10.3892/ol.2016.4327

[6] Ishikawa, T., Suzuki, A. and Yasui, N. (1990) Distal Posterior Inferior Cerebellar Aneurysms: Report of 12 Cases. Neurologia Medico-Chirurgica (Tokyo), 30, 100-108. https://doi.org/10.2176/nmc.30.100

[7] Yasargil, M.G. (1984) Vertebrobasilar Aneurysms. In: Microneurosurgery, Vol. 2: Clinical Considerations, Surgery of the Intracranial Aneurysms and Results, Georg Thieme, Stuttgart, 232-295.

[8] Sedat, J., Chau, Y., Mahagne, M.H., Bourg, V., Lonjon, M. and Paquis, P. (2007) Dissection of the Posteroinferior Cerebellar Artery: Clinical Characteristics and Long-Term Follow-Up in Five Cases. Cerebrovascular Diseases, 24, 183-190. https://doi.org/10.1159/000104475

[9] Wu, J., Xu, F., Yu, Z.Q., Zhou, Y.X., Cui, G., Li, X.D., Zhou, D., Zhang, S.M. and Wang, Z. (2012) Clinical Experiences of Ruptured Posteroinferior Cerebellar Artery Aneurysms and Anatomical Analysis in the Cadaver in a Single Center of China. Clinical Neurology and Neurosurgery, 114, 366-371.

https://doi.org/10.1016/j.clineuro.2011.11.007

[10] Horiuchi, T., Tanaka, Y., Hongo, K., Nitta, J., Kusano, Y. and Kobayashi, S. (2003) Characteristics of Distal Posteroinferior Cerebellar Artery Aneurysms. Neurosurgery, 53, 589-596. https://doi.org/10.1227/01.NEU.0000079493.50657.1D

[11] Lewis, S.B., Chang, D.J., Peace, D.A., Lafrentz, P.J. and Day, A.L. (2002) Distal Posterior Inferior Cerebellar Artery Aneurysms: Clinical Features and Management. Journal of Neurosurgery, 97, 756-766. https://doi.org/10.3171/jns.2002.97.4.0756

[12] Pilipenko, Y., Eliava, S., Okishev, D., Okisheva, E. and Spyrou, A. (2019) Vertebral Artery and Posterior Inferior Cerebellar Artery Aneurysms: Results of Microsurgical Treatment of Eighty Patients. Surgical Neurology International, 10, 227. https://doi.org/10.25259/SNI 3262019

[13] Horowitz, M., Kopitnik, T., Landreneau, F., Krummerman, J., Batjer, H.H., Thomas, G. and Samson, D. (1998) Posteroinferior Cerebellar Artery Aneurysms: Surgical Results for 38 Patients. Neurosurgery, 43, 1026-1032. https://doi.org/10.1097/00006123-199811000-00012

[14] Al-khayat, H., Al-Khayat, H., Beshay, J., Manner, D. and White, J. (2005) Vertebral Artery-Posteroinferior Cerebellar Artery Aneurysms: Clinical and Lower Cranial Nerve Outcomes in 52 Patients. Neurosurgery, 56, 2-11. https://doi.org/10.1227/01.NEU.0000145784.43594.88 
[15] Lemole Jr., G.M., Henn, J., Javedan, S., Deshmukh, V. and Spetzler, R.F. (2002) Cerebral Revascularization Performed Using Posterior Inferior Cerebellar Artery-Posterior Inferior Cerebellar Artery Bypass: Report of Four Cases and Literature Review. Journal of Neurosurgery, 97, 219-223. https://doi.org/10.3171/jns.2002.97.1.0219

[16] Yoon, S.-M., Shim, J.-J., Kim, S.-H. and Chang, J.-C. (2012) Bilateral Vertebral Artery Dissecting Aneurysms Presenting with Subarachnoid Hemorrhage Treated by Staged Coil Trapping and Covered Stents Graft. Journal of Korean Neurosurgical Society, 51, 155-159. https://doi.org/10.3340/jkns.2012.51.3.155 